\title{
DEXH-Box protein DHX30 is required for optimal function of the zinc-finger antiviral protein
}

\author{
Peiying Ye ${ }^{1,2}$, Shufeng Liu ${ }^{1}$, Yiping Zhu ${ }^{1,2}$, Guifang Chen ${ }^{1}$, Guangxia Gao ${ }^{1 凶}$ \\ ${ }^{1}$ Center for Infection and Immunity, Institute of Biophysics, Chinese Academy of Sciences, Beijing 100101, China \\ ${ }^{2}$ Graduate School of Chinese Academy of Sciences, Beijing 100039, China \\ \ Correspondence: gaogx@moon.ibp.ac.cn
}

Received August 29, 2010 Accepted September 26, 2010

\begin{abstract}
The zinc-finger antiviral protein (ZAP) is a host factor that specifically inhibits the replication of certain viruses by eliminating viral mRNAs in the cytoplasm. In previous studies, we demonstrated that ZAP directly binds to the viral mRNAs and recruits the RNA exosome to degrade the target RNA. In this article, we provide evidence that a DEXH box RNA helicase, DHX30, is required for optimal antiviral activity of ZAP. Pull-down and co-immunoprecipitation assays demonstrated that $\mathrm{DHX30}$ and ZAP interacted with each other via their $\mathbf{N}$ terminal domains. Downregulation of DHX30 with shRNAs reduced ZAP's antiviral activity. These data implicate that DHX30 is a cellular factor involved in the antiviral function of ZAP.
\end{abstract}

KEYWORDS zinc-finger antiviral protein, RNA helicase, $\mathrm{DHX} 30$

\section{INTRODUCTION}

The zinc-finger antiviral protein (ZAP) was originally cloned as a host factor that prevented cells from infection by Moloney murine leukemia virus (MLV) (Gao et al., 2002). Overexpression of ZAP inhibits the replication of MLV, Ebola virus and Marburg virus (Muller et al., 2007), and several members of the Alpha virus genus (Bick et al., 2003). The mRNA level of endogenous ZAP in mouse bone marrowderived dendritic cells can be significantly up-regulated by interferon treatment or Sindbis virus (SINV) infection (Zhang et al., 2007). These results suggest that ZAP may play an important role in the host antiviral system in vivo.

ZAP specifically eliminates the cytoplasmic viral mRNA
(Gao et al., 2002). It directly binds to the target viral mRNAs through the zinc-finger motifs (Guo et al., 2004) and recruits the RNA degradation machineries to degrade the target viral mRNA (Guo et al., 2007; Zhu and Gao, 2008). mRNA degradation is a highly organized and complex process (Guhaniyogi and Brewer, 2001; Garneau et al., 2007), involving many co-factors. We previously reported that the DEAD-box RNA helicase p72 interacted with ZAP and was required for the optimal function of ZAP (Chen et al., 2008). It is reasonable to speculate that more co-factors may be involved in the antiviral function of ZAP.

$\mathrm{DHX} 30$ is an RNA helicase belonging to the $\mathrm{DExD} / \mathrm{H}$ family since it bears the DEVH signature sequence in the Walker $B$ motif (Zhou et al., 2007). There are three isoforms of DHX30 reported on NCBI. Isoform 1 and 2 contain the DEVH signature helicase domain and a putative type $A$ dsRBD at the N-terminal region. Isoform 3 (NM_138614.1) lacks the helicase core domain and is believed to exist at very low abundance because it is a nonsense-mediated mRNA decay (NMD) candidate. Annotation of AceView suggests that DHX30 gene contains 39 different introns. Furthermore, transcription of DHX30 produces 22 different mRNAs by alternative splicing, which have good potential to be translated into 18 different isoforms. Indeed, besides Isoform 1,2 and 3 , a specific isoform of DHX30 was identified as a mitochondrial nucleoid protein. The antibody raised against the N-terminal domain of DHX30 detects both nuclear and mitochondrial isoforms of DHX30 (Wang and Bogenhagen, 2006). It has been reported that overexpression of DHX30 enhanced HIV-1 gene expression by augmenting basal transcription from the HIV-1 LTR promoter. However, knockdown of DHX30 did not distinctly affect HIV-1 LTR-dependent transcription (Zhou et al., 2007). Moreover, overexpression of 
DHX30 severely restrained the packaging of HIV-1 RNA, and thereby reduced virus infectivity (Zhou et al., 2007).

In the present study, we identified DHX30 as a ZAPinteracting protein involved in the antiviral function of ZAP.

\section{RESULTS}

\section{Identification of the DHX30 RNA helicase as a ZAP-interacting protein}

To further study the mechanism for ZAP's antiviral activity, we set out to identify cellular factors that associated with ZAP. Hismyc-tagged rat ZAP (rZAP) was expressed in 293TRex-ZAP cells in a tetracycline-inducible manner. $r Z A P$ and its associated proteins were purified using Ni-NTA Agarose, resolved on SDS/PAGE and visualized by Coomassie blue staining. To prevent possible nonspecific RNA tethering, the lysates were treated with RNase A. Compared with the precipitates of 293TRex control cells, a specific band of $130 \mathrm{kD}$ was detected in the rZAP-expressing cells (Fig. 1A). This band was excised and subjected to matrix-assisted laser desorption ionization time-of-flight (MALDI-TOF) mass spectrometry (MS) analysis to identify the protein. The result of database search identified the DHX30 RNA helicase as a candidate.

To confirm the interaction between rZAP and DHX30, coimmunoprecipitation assays were performed. Flag-tagged rZAP and myc-tagged DHX30 were co-transfected into HEK293T cells. Immunoprecipitation of DHX30 using the anti-myc antibody coprecipitated Flag-tagged rZAP in the absence of RNase A (Fig. 1B). Treatment of the cell lysates with RNase $A$ reduced the interaction between $\mathrm{rZAP}$ and $\mathrm{DHX30}$. The reduced interaction was likely due to the fact that DHX30 precipitated easily after RNase A treatment, leaving relatively low amount of $\mathrm{DHX} 30$ in the supernatant for immunoprecipitation (Fig. 1B, lower panel). In addition, it is also possible that both RNA-dependent and independent ZAP-DHX30 interactions may exist and that RNase treatment may have removed the RNA-dependent interaction. The RNA-independent interaction between ZAP and DHX30 was further confirmed in later experiments (see below). In a reverse experiment, immunoprecipitation of myc-tagged rZAP coprecipitated DHX30 in the absence or presence of RNase A (Fig. 1C). The interaction between human ZAP (hZAP) and DHX30 was also investigated and as expected, hZAP also interacted with DHX30 (Fig. 1D).

\section{Mapping the binding domains of ZAP and DHX30}

The N-terminal domain of ZAP fused with the zeocin resistance gene (NZAP-Zeo) displayed the same antiviral activity as the full-length ZAP (Gao et al., 2002). If DHX30 is involved in the function of ZAP, it would be expected to interact with the $\mathrm{N}$-terminal domain of ZAP. The $\mathrm{N}$-terminal domain of rZAP (rNZAP254), the C-terminal domains of rZAP
(rCZAP193 and rCZAP236) were individually co-expressed with $\mathrm{DHX} 30$ and analyzed for their interactions with DHX30. As expected, immunoprecipitation of DHX30 coprecipitated only the N-terminal domain of rZAP, but not the C-terminal domain (Fig. 2A). In the reverse experiment, only immunoprecipitation of the $\mathrm{N}$-terminal domain of rZAP, but not the $\mathrm{C}$ terminal domains of rZAP, coprecipitated DHX30 (Fig. 2B). These results established that $\mathrm{DHX} 30$ interacted with the $\mathrm{N}$ terminal domain of rZAP.

To determine the domains of $\mathrm{DHX} 30$ responsible for its interaction with ZAP, DHX30 truncation mutants were constructed and analyzed for their abilities to bind to ZAP (Fig. 3). Immunoprecipitation of $\mathrm{N}$-terminal domain of $\mathrm{DHX} 30$ (NDHX30) coprecipitated rZAP (Fig. 3A). The truncation mutant containing the $\mathrm{N}$-terminal domain and the core domain $(\mathrm{N}+$ core) also interacted with $\mathrm{rZAP}$, although the band of coimmunoprecipitated ZAP by $\mathrm{N}+$ core was weaker compared with the $\mathrm{N}$-terminal domain alone. The weaker band likely reflected the relatively low level of $\mathrm{N}+$ core (Fig. $3 \mathrm{~A}$, lower panel) since the intensities of the bands are roughly proportional. In contrast, the core domain alone or the Cterminal domain of DHX30 failed to interact with rZAP (Fig. $3 A$ ). The interaction between the $\mathrm{N}$-terminal domain of DHX30 and rZAP was not affected by the treatment of the cell lysate with RNase A (Fig. 3B). Pull-down assays were employed to further prove the interaction between the $\mathrm{N}$ terminal domain of $\mathrm{DHX} 30$ and ZAP using bacterially expressed NDHX30 fused with maltose binding protein (MBP). With or without RNase A treatment, MBP-NDHX30 pulled down rZAP, hZAP and rNZAP (Fig. 3C). The direct interaction between NDHX30 and hNZAP was also confirmed by pull-down assays using purified bacterially expressed proteins (Fig. 3D).

\section{Downregulation of DHX30 reduced ZAP's activity}

To investigate the role of DHX30 in ZAP's antiviral function, three shRNAs directed against DHX30 were designed and validated. The plasmids expressing the shRNAs directed against $\mathrm{DHX} 30$ (shDHX30i) were first tested for their ability to down-regulate the expression of $\mathrm{DHX} 30$ by cotransfected into 293A cells with the construct expressing the myc-tagged DHX30. Compared with the control shRNA (ctrl), the shRNAs directed against DHX30 (Di-1, Di-2 and Di-3) significantly reduced the expression level of myc-tagged DHX30 (Fig. 4A). The efficiency of these three shRNAs against endogenous DHX30 was confirmed by RT-PCR (Fig. 4B). To test the effect of DHX30 downregulation on the activity of ZAP, 293TRexZAP cells were cotransfected with pMLV-Luc reporter and shDHX30i, and assayed for inhibition of the reporter. The shRNA directed against p72 (72i), which has been shown to reduce ZAP's activity, was used as a positive control. All three shRNAs against DHX30 reduced the activities of both rZAP and hZAP (Fig. 4C and 4D). 
A

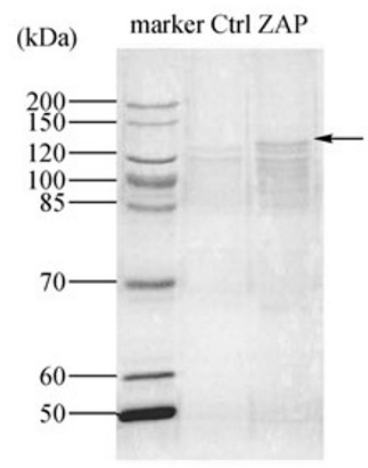

B

\begin{tabular}{|c|c|c|c|c|c|c|c|c|c|c|}
\hline & \multicolumn{4}{|c|}{$2 \%$ input } & \multicolumn{6}{|c|}{ IP: $\alpha$-myc } \\
\hline rZAP-Flag & - & + & + & + & - & - & + & + & + & + \\
\hline DHX30-myc & $+\quad+$ & $+\quad+$ & - & - & + & + & + & + & - & - \\
\hline RNase A & $-\quad+$ & $-\quad+$ & - & + & - & + & - & + & - & + \\
\hline rZAP-Flag & & $\infty$ & - & 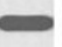 & & & & - & & \\
\hline
\end{tabular}

C

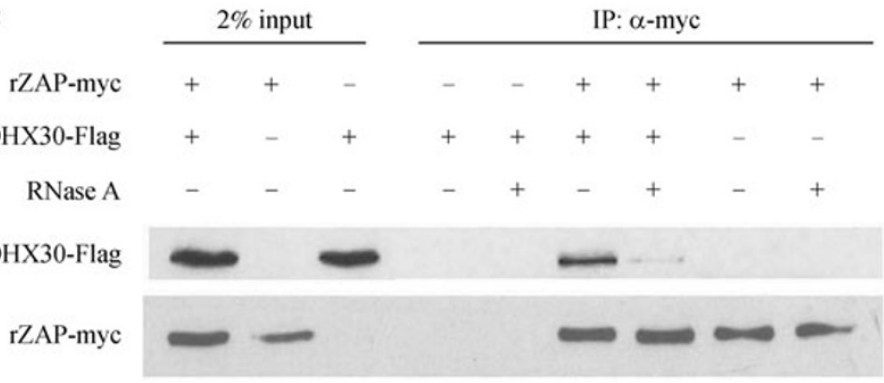

D

\begin{tabular}{|c|c|c|c|c|c|c|}
\hline \multirow[b]{2}{*}{ hZAP-myc } & \multicolumn{3}{|c|}{$2 \%$ input } & \multicolumn{3}{|c|}{ IP: $\alpha$-Flag } \\
\hline & - & + & + & - & + & + \\
\hline HX30-Flag & + & + & - & + & + & - \\
\hline RNase A & + & + & + & $\begin{array}{llll}+ & & l & 0\end{array}$ & + & + \\
\hline hZAP-myc & & & & & - & \\
\hline
\end{tabular}

Figure 1. DHX30 interacted with ZAP. (A) Identification of DHX30 as a putative ZAP-interacting protein 293TRex or 293TRexZAP cells were treated with tetracycline to induce ZAP expression. ZAP and associated proteins were purified by using Ni-NTA Agarose and resolved on $10 \%$ SDS-PAGE. The proteins were visualized by Coomassie blue staining. The band specific to the ZAPexpressing cells (indicated by the arrow) was excised and subjected to MALDI-TOF mass spectrometry analysis. Ctrl: 293TRex control cells; ZAP: ZAP-expressing 293TRex-ZAP cells. (B) co-IP of rZAP with DHX30. The plasmids expressing Flag-tagged rZAP and myc-tagged DHX30 were transiently cotransfected into HEK293T cells. At $48 \mathrm{~h}$ posttransfection, the cells were lysed. The cell lysates were immunoprecipitated with the anti-myc antibody in the presence (+) or absence (-) of RNase A and Western blotted with the anti-Flag and anti-myc antibodies. (C) co-IP of DHX30 with rZAP. The plasmids expressing Flag-tagged DHX30 and myc-tagged rZAP were transiently cotransfected into HEK293T cells. At $48 \mathrm{~h}$ posttransfection, the cells were lysed. The cell lysates were immunoprecipitated with the anti-myc antibody in the presence $(+)$ or absence $(-)$ of RNase A and Western blotted with the anti-Flag and anti-myc antibodies. (D) co-IP of DHX30 with hZAP. The plasmids expressing Flag-tagged DHX30 and myc-tagged hZAP were transiently cotransfected into HEK293T cells. The cell lysates were immunoprecipitated with the anti-Flag antibody in the presence $(+)$ of RNase A and Western blotted with the anti-myc and anti-Flag antibodies. 
A

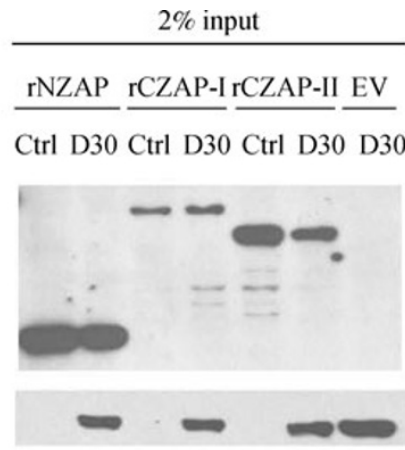

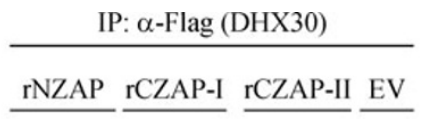

Ctrl D30 Ctrl D30 Ctrl D30 D30

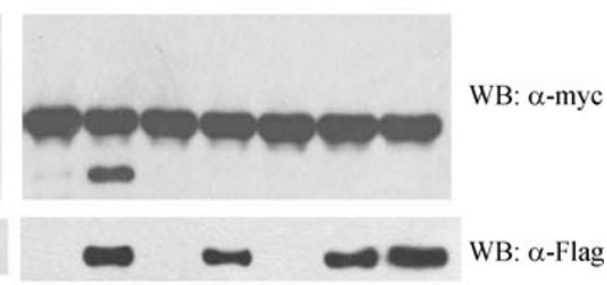

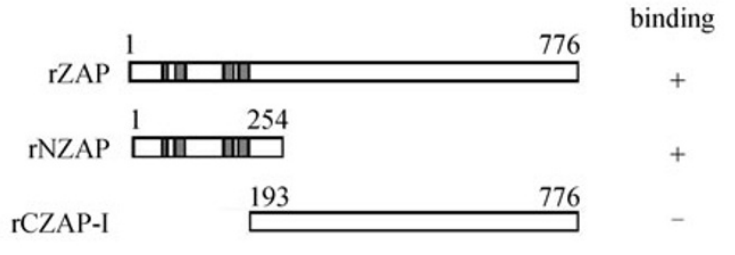

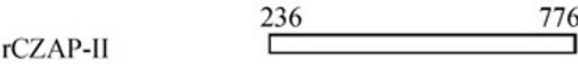

B

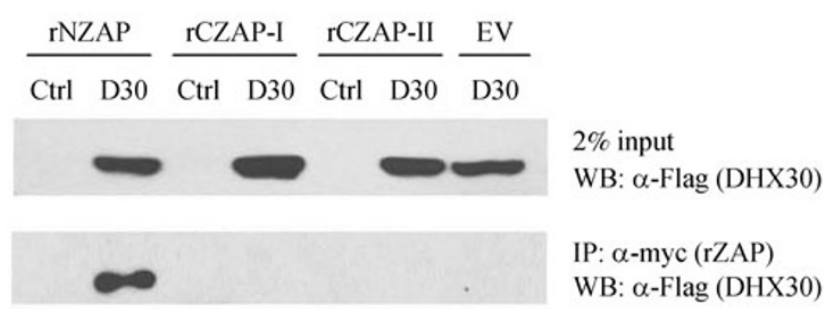

Figure 2. Mapping the binding domains of ZAP. (A) (B) co-IP of rZAP truncation mutants with DHX30. (A) The plasmid expressing the indicated myc-tagged truncated rZAP protein was individually cotransfected into HEK293T cells with the plasmid expressing Flag-tagged DHX30. The cell lysates were immunoprecipitated with the anti-Flag antibody in the presence of RNase A and Western-blotted with the anti-myc and anti-Flag antibodies. Input, total cell lysate; Ctrl, pCMV-HA-Flag empty vector; EV, pcDNA4 empty vector; D30, DHX30. Schematic representations of the rZAP proteins are shown, and their binding activities are summarized. (B) The plasmid expressing the indicated myc-tagged truncated rZAP protein was individually cotransfected into HEK293T cells with the plasmid expressing Flag-tagged DHX30. The cell lysates were immunoprecipitated with the anti-myc antibody in the presence of RNase A and Western-blotted with the anti-Flag and anti-myc antibodies. Ctrl, pCMV-HA-Flag empty vector; EV, pcDNA4 empty vector; D30, DHX30.

In an attempt to test whether DHX30 is also involved in other RNA degradation processes, DHX30i was analyzed for its effect on the degradation of AU-rich element (ARE)containing mRNA by AUBP, which binds to ARE and recruits exosome to degrade the ARE-containing RNA. DHX30i had little effect on ARE-containing reporter (Fig. 4E), suggesting that DHX30 RNA helicase is not involved in ARE mediated mRNA decay.

\section{DISCUSSION}

ZAP specifically inhibits the replication of certain viruses by recognizing the ZAP-responsive element (ZRE) present in the target viral mRNAs and recruiting the cellular RNA degradation machineries to degrade the target viral mRNAs. Among the ZREs so far identified, no obvious sequence similarity or common motifs has been observed. The only common feature of these ZREs is that they are at least 500 nucleotides long. It is plausible that the ZRE-containing viral mRNAs form complex tertiary structures in vivo. Studies of reconstituted RNA exosome revealed that the exosome could process only extended RNA substrate but not stem-loop structured RNA substrate (Liu et al., 2006). Thus, it is reasonable to speculate that disruption of the secondary structures by RNA helicases is necessary for efficient ZAP-mediated viral mRNA degradation. 


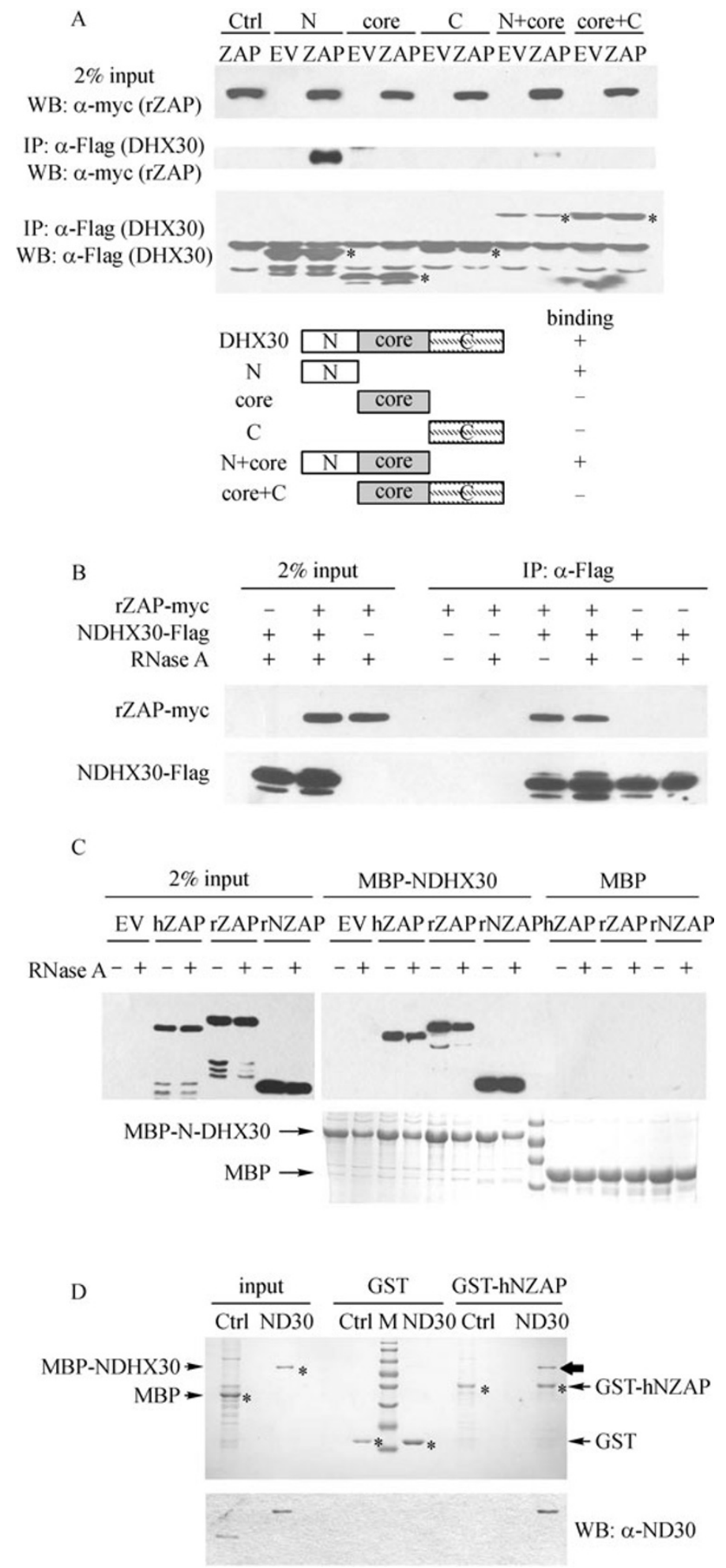

Figure 3. Mapping the binding domains of DHX30. (A) co-IP of rZAP with DHX30 truncation mutants. The plasmid expressing the indicated Flag-tagged truncated DHX30 protein was individually cotransfected into HEK293T cells with the plasmid expressing myc-tagged rZAP. The cell lysates were immunoprecipitated with the anti-Flag antibody in the presence (+) or absence (-) of RNase $A$ and Western-blotted with the anti-myc and anti-Flag antibodies. Input, total cell lysate; Ctrl, pCMV-HA-Flag; EV, pcDNA4. The positions of the truncated $\mathrm{DH} \times 30$ proteins are indicated by asterisks. Schematic representations of the $\mathrm{DH} \times 30$ proteins are shown, 
Defined by the highly conserved sequence motifs required for RNA binding and ATP hydrolysis, most RNA helicases have the intrinsic capability to unwind RNA duplexes (Jankowsky and Fairman, 2007). Furthermore, variable Nor C-terminal domain flanking the highly conserved helicase core usually grants unique substrate specificity. It is conceivable that multiple RNA helicases may be needed to unwind complex RNA tertiary structure. We previously reported that the DEAD box RNA helicase $p 72$ was required for the optimal function of ZAP. Here, we observed that $\mathrm{DHX} 30$ interacted with ZAP through its $\mathrm{N}$-terminal domain, and downregulation of DHX30 reduced ZAP's antiviral activity. Whether and how these two RNA helicases coordinate to contribute to ZAP's activity awaits further investigation.

It is unclear yet how DHX30 is involved in ZAP-mediated antiviral activity. The likely possibility is that ZAP binds to the target RNA and recruits DHX30 to help unwind the RNA to facilitate efficient RNA degradation. However, other possibilities also exist. For example, ZAP may recruit DHX30 just as a co-factor to further recruit other RNA degradation machineries. Further investigation is needed to fully understand how DHX30 is involved in the antiviral activity of ZAP.

\section{MATERIALS AND METHODS}

\section{Plasmids}

The plasmids pcDNA4TO/myc-ZAP, pcDNA4TO/myc-NZAP, pcDNA4TO/myc-CZAP-A, and pcDNA4TO/myc-CZAP-C, which express myc-tagged full-length rZAP, rNZAP (amino acids 1-254), rCZAP-I (amino acids 193-776), and rCZAP-II (amino acids 236-776), respectively, have been described previously (Guo et al., 2007). pcDNA4TO-ZAP-Flag expresses Flag-tagged ZAP. The coding sequence of rZAP was amplified from pcDNA4TO/myc-ZAP by using forward primer ZAP- 5 bearing a Kpnl site and reverse primer ZAP-3 bearing a Notl site, and cloned into the expression vector pcDNA3-Flag by using these two sites: ZAP-5: 5'-GGGGTACCATGGCAGATCCCGGGGTATGCTGTTTC-3' ; ZAP3: 5'-ATAAGAATGCGGCCGCTCTGGACCTCTTCTCTTCTGC-3'.

pcDNA4TO/myc-hZAP expresses myc-tagged human ZAP isoform 2. The coding sequence of human ZAP iso2 was amplified from a human fetal liver cDNA library by using forward primer hZAP-up bearing an BamHI site and reverse primer hZAP-down bearing a Notl site and cloned into the expression vector pcDNA4TO/myc-his by using these two sites: hZAP-up: 5'-AATAGGATCCGCCACCATGGCGGACCCGGAGGTGTGC-3' and hZAP-down: 5'-ATCTGAGCGGCCGCGGTCTGGCCCTCTCTTCATCTGCT-3'.

The DHX30-deletion mutants were generated by cloning PCRderived DHX30 fragments into pCMV-HA-Flag (Guo et al., 2007). An EcoRI site was built in the forward primers, and a Notl site was built in the reverse primers. The primers are listed with the restriction sites italicized:

DHX30-FP2, 5'-GGAATTCGCAGCTTCTAGGGACCTATTAAA-3';

DHX30-RP2,5'-ATATAGGCGGCCGCTCAGTCGTCAGCTGTCTT GCG-3';

DHX30N-RP, 5'-ATATAGGCGGCCGCTCATGGGTCCACAGGTA GCTG-3';

DHX30-core-FP, 5'-GGAATTCCATCGGGACACCATCCTCA-3';

DHX30-core-RP, 5'-ATATAGGCGGCCGCTCATGTGATGGAAGT CTCAGC-3';

DHX30C-FP, 5'-GGAATTCATCAATGACATCGTGCATG-3'.

The plasmid-expressing N-terminal portion of hZAP with GST fused at the $\mathrm{N}$-terminus in $\mathrm{E}$. coli has been described previously (Law et al., 2010). The plasmid expressing the N-terminal portion of DHX30 with MBP fused at the $\mathrm{N}$-terminus in E.coli was generated by cloning PCR-derived DHX30 fragments into pMAL-c2x-linker vector. An EcoRI site was built in the forward primers, MBP-DHX30-FP (5'GGAATTCGCAGCTTCTAGGGACCTATTAAA-3') and a Notl site was built in the reverse primers DHX30N-RP.

pSUPER.puro-DHX30 RNAi expresses shRNA directed against DHX30. Oligonucleotides DHX30-RNAi FP and DHX30-RNAi RP were annealed and cloned into pSUPER.puro vector by using the Bglll and HindlII sites to generate pSUPER.puro-DHX30 RNAi. pSUPER.puro control RNAi and GFP RNAi were constructed by using the same strategy. The sequences of the oligos are listed below:

DHX30 RNAi-1-FP: 5'-GATCCCCCAGCTGAATCCAGAGAGTATTCAAGAGATACTCTCTGGATTCAGCTGTTTTTA-3'; DHX30 RNAi-1-RP: 5'-AGCTTAAAAACAGCTGAATCCAGAGAGTATCTCTTGAATACTCTCTGGATTCAGCTGGGG-3';

DHX30 RNAi-2-FP: 5'-GATCCCCGCTGTGGACAGTCCAAACATTCAAGAGATGTTTGGACTGTCCACAGCTTTTTA-3';

DHX30 RNAi-2-RP:5'-AGCTTAAAAAGCTGTGGACAGTCCAAACATCTCTTGAATGTTTGGACTGTCCACAGCGGG-3';

DHX30 RNAi-3-FP: 5'-GATCCCCGATGGATCAGAAGGCCATATTCAAGAGATATGGCCTTCTGATCCATCTTTTTA-3';

and their binding activities are summarized. (B) co-IP of rZAP with N-terminal domain of DHX30. The plasmids expressing Flagtagged NDHX30 and myc-tagged rZAP were transiently cotransfected into HEK293T cells. The cell lysates were immunoprecipitated with the anti-Flag antibody in the presence $(+)$ or absence $(-)$ of RNase A and Western blotted with the anti-myc and anti-Flag antibodies. (C) Pull-down of ZAP with MBP-N-DHX30. Bacterially expressed MBP or MBP-NDHX30 was immobilized onto Amylose resin and incubated with the lysates of the cells expressing the indicated myc-tagged ZAP proteins in the presence of RNase $A$ at $4{ }^{\circ} \mathrm{C}$ for $2 \mathrm{~h}$. The resins were washed and boiled in the sample loading buffer. The proteins were resolved by SDS-PAGE and detected by Western blotting using the anti-myc antibody. Input, total cell lysate; EV, pcDNA4. The positions of MBP and MBP-NDHX30 are indicated by arrows. (D) Pull-down of MBP-N-DHX30 with GST-N-hZAP. Bacterially expressed GST or GST-N-hZAP was immobilized onto glutathione-Sepharose $4 \mathrm{~B}$ resin and incubated with purified MBP-NDHX30 in the presence of RNase $A$ at $4^{\circ} \mathrm{C}$ for $2 \mathrm{~h}$. The resins were washed and boiled in the sample loading buffer. The proteins were resolved by SDS-PAGE and detected by Coomassie staining (upper panel) or Western blotting using the anti-ND30 antibody (lower panel). Input, purified fusion proteins; Ctrl, MBP; ND30, MBP$\mathrm{NDHX} 30$. The positions of the fusion proteins are indicated by asterisks. The band of the pull-downed protein is indicated by an arrow. 
A

Ctrl 1 Ctrl $2 \quad$ Di-1 Di-2 Di-3

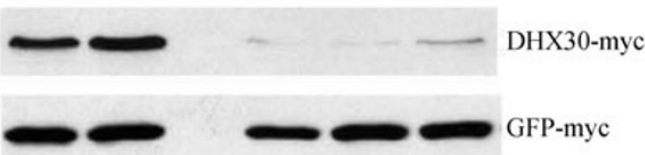

B
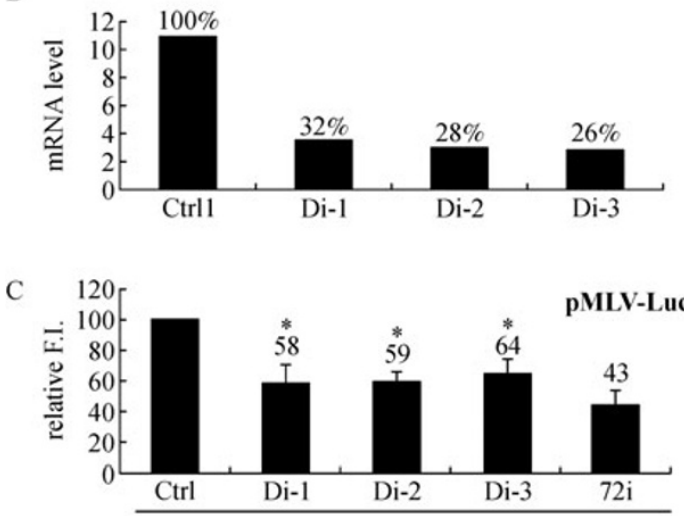

293TRex-rZAP cells

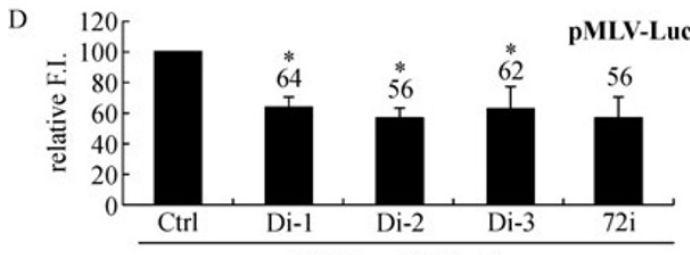

293TRex-rZAP cells

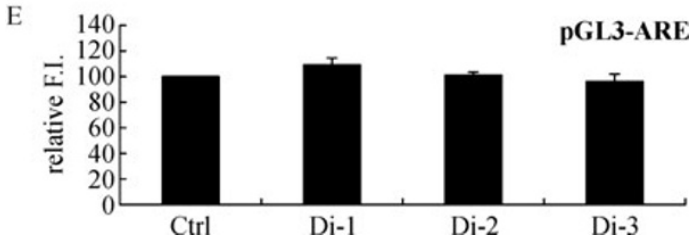

Figure 4. Downregulation of DHX30 reduced ZAP's activity. (A) The effect of DHX30 shRNA on DHX30 expression. The plasmids expressing myc-tagged DHX30 and GFP was cotransfected into HEK293A cells with the plasmid expressing control shRNA (Ctrl1, Ctrl2) or the shRNA directed against DHX30 (Di-1, Di-2 or Di-3). The expression levels of the DHX30 proteins were measured by Western blotting using the anti-myc antibody. GFP-myc served as an internal control. (B) The effect of DHX30 shRNA on the mRNA level of endogenous DHX30. The plasmid expressing the indicated shRNA was transfected into HEK293 cells. The cells were selected in puromycin to remove the untransfected cells. At $48 \mathrm{~h}$ after puromycin treatment, total RNA was isolated and the RNA level of endogenous DHX30 was measured by Realtime PCR using GAPDH as an internal control. (C) (D) The effect of DHX30 shRNA on ZAP's activity. The pMLV-Luc reporter was cotransfected with the plasmid expressing the indicated shRNA into (C) 293TRex-rZAP cells or (D) 293TRex-hZAP cells. At $4 \mathrm{~h}$ posttransfection, the cells were treated with puromycin to remove the untransfected cells and tetracycline was added to induce ZAP expression. At $48 \mathrm{~h}$ posttransfection, the cells were lysed and luciferase activities were measured. Fold inhibition was calculated as the luciferase activity in the mock-treated cells divided by the luciferase activity in the tetracycline-treated cells. Relative fold inhibition was calculated as percentage of the fold inhibition in the presence of the Ctrl RNAi (average of Ctrl1 and Ctrl2) divided by the fold inhibition in the presence of the indicated RNAi. 72i served as a positive control. The data are means $+S E$ of four independent experiments. The asterisks $\left(^{*}\right)$ denote $P<0.001$. (E) The effect of shDHX30 on AUBP-mediated mRNA degradation. The ARE-containing reporter, pGL3-ARE-Luc, or pGL3 empty vector was cotransfected with the plasmid expressing the indicated shRNA into HEK293 cells. At $48 \mathrm{~h}$ posttransfection, the cells were lysed and luciferase activities were measured. Fold inhibition was calculated as the luciferase activity in the cells transfected with pGL3 divided by the luciferase activity in the cells transfected with pGL3-ARE-Luc. Relative fold inhibition was calculated as percentage of the fold inhibition in the presence of the Ctrl RNAi (average of Ctrl1 and Ctrl2) divided by the fold inhibition in the presence of the indicated RNAi. The data are means + SE of three independent experiments. 
DHX30 RNAi-3-RP: 5'-AGCTTAAAAAGATGGATCAGAAGGCCATATCTCTTGAATATGGCCTTCTGATCCATCGGG-3';

Control RNAi-FP: 5'-GATCCCCGAGCACTCTGAACTACCTGTTCAAGAGACAGGTAGTTCAGAGTGCTCTTTTTGGAAA-3';

Control RNAi-RP: 5'-AGCTTTTCCAAAAAGAGCACTCTGAACTACCTGTCTCTTGAACAGGTAGTTCAGAGTGCTCGGG-3'.

\section{Cell culture}

All the cells were maintained in DMEM supplemented with $10 \%$ FBS. Transfection was performed by using Lipofectamine 2000 (Invitrogen) following the manufacturer's instructions. 293TRex and 293TRexZAP cell lines have been described previously (Guo et al., 2004). To analyze the effect of the RNAi on ZAP's activity, 293TRex-ZAP cells were transfected with the pMLV-Luc reporter, $\mathrm{pRL}$-TK (for normalizing transfection efficiency) and the effector-expressing plasmid. Immediately after transfection, the cells were mock-treated or treated with tetracycline to induce ZAP expression. Forty-eight hours after transfection, luciferase activities were measured and normalized by dividing the firefly luciferase activity with the Renilla luciferase activity. Fold inhibition by ZAP was calculated as the normalized luciferase activity in the mock-treated cells divided by the normalized luciferase activity in the tetracycline-treated cells. To assay the effect of RNAi on the activity of AUBP, 293A cells were cotransfected with pGL3 or a type II ARE-containing reporter, pGL3-ARE-Luc (Guo et al., 2004), together with $\mathrm{pRL}-\mathrm{TK}$, and the shRNA-expressing plasmid. At $48 \mathrm{~h}$ posttransfection, the luciferase activities were measured. Fold inhibition by AUBP was calculated as the normalized luciferase activity in the pGL3-transfected cells divided by the normalized luciferase activity in the pGL3-ARE-Luc-transfected cells. The statistical significance of the data is analyzed with an SPSS program.

\section{Identification of ZAP-interacting proteins}

293TRex-ZAP cells were treated with tetracycline at a final concentration of $1 \mu \mathrm{g} / \mathrm{mL}$ to induce ZAP expression. Thirty-six hours after induction, the cells were lysed with lysis buffer $B$ [ $30 \mathrm{mM}$ Hepes ( $\mathrm{pH}$ 7.6), $100 \mathrm{mM} \mathrm{NaCl}, 0.5 \%$ Nonidet P-40, and protease inhibitors mixture]. The lysates were clarified by centrifugation at $13,000 \mathrm{rpm}$ for $10 \mathrm{~min}$ at $4^{\circ} \mathrm{C}$ in a microcentrifuge (Sorvall Fresco), treated with $50 \mu \mathrm{g} / \mathrm{mL}$ RNase A for $15 \mathrm{~min}$ at $37^{\circ} \mathrm{C}$. ZAP and associated proteins were purified by using Ni-NTA Agarose (QIAGEN) following the manufacturer's instructions. The ZAP complex was eluted with $100 \mathrm{mM}$ imidazole and resolved on $10 \%$ acrylamide gels for SDSPAGE. The proteins were visualized by Coomassie blue staining. The specific bands from the ZAP-expressing cells were excised from the gel and digested with trypsin. The resulting peptides were analyzed by MALDI-TOF MS. The acquired MS data were analyzed with a human nucleotide/protein database by using a MASCOT database search tool for peptide identification. The protein was identified when multiple peptides corresponding to the ORF of a protein were identified.

\section{RNA isolation, reverse transcription and realtime-PCR}

Total RNA was isolated from HEK293A cells using the RNeasy kit (Qiagen) following the manufacturer's protocol and checked for its quality and concentration. $2 \mu \mathrm{g}$ of total RNA was reverse transcribed using the M-MLV reverse transcriptase. The cDNA was amplified by using $1 \mu \mathrm{L}$ of the $\mathrm{RT}$ reaction into a $20-\mu \mathrm{L}$ PCR reaction containing 10 pmol of the following primers: DHX30 realtime FP 5'-GCACAAGTCGACCATTAACAGGGAG-3' and DHX30 realtime RP 5'ACTGTCGCTCAGTGAGATGGTGGCC-3'. Amplification was carried out under standard conditions using $2 \times$ PCR Master Mix (Tiangen Biotech, Beijing, China) on the Rotor-Gene $6000^{\mathrm{TM}}$ real-time PCR instrument (Corbett Research).

\section{Antibodies}

The anti-myc mouse monoclonal antibody 9E10 (Santa Cruz Biotechnology) was used to immunoprecipitate or detect proteins with the myc epitope, and the anti-Flag mouse monoclonal antibody M2 (Sigma-Aldrich) was used to immunoprecipitate or detect proteins with the Flag epitope. Bacterially expressed $\mathrm{N}$-terminal portion of $\mathrm{DHX} 30$ was used to immunize rabbits to generate polyclonal antibodies against $\mathrm{DH} \times 30$. The antibodies were affinity-purified by using the cognate protein.

\section{Coimmunoprecipitation}

Cells were lysed in lysis buffer B [30 mM Hepes (pH 7.6), 100 mM $\mathrm{NaCl}, 0.5 \%$ Nonidet P-40, and protease inhibitors mixture] mocktreated or treated with RNase A $(5 \mathrm{mg} / \mathrm{mL})$ on ice for more than one hour, and the lysates were clarified by centrifugation at $4^{\circ} \mathrm{C}$ for $20 \mathrm{~min}$ at $13,000 \mathrm{rpm}$. The supernatant was mixed with protein $\mathrm{G}$ plus agarose (Santa Cruz Biotechnology) and the antibody and incubated at $4^{\circ} \mathrm{C}$ for $2 \mathrm{~h}$. The resins were then washed three times with lysis buffer $\mathrm{B}$, and the bound proteins were detected by Western blotting.

\section{ACKNOWLEDGEMENTS}

We thank Jing Sun and Yihui Xu for technical support and Dr. Fuquan Yang for help with the MALDI-TOF analyses. This work was in part supported by the grant to Guangxia Gao from National Science Foundation of China (Grant No. 81030030), and by the grant to Guifang Chen from National Science Foundation (Grant No. 30800053).

\section{ABBREVIATIONS}

AMD, ARE mediated mRNA decay; Ctrl, control plasmid; Di-1, shRNA directed against DHX30i number 1; Di-2, shRNA directed against DHX30i number 2; Di-3, shRNA directed against DHX30i number 3; dsRBD, double-stranded RNA-binding domain; EV, empty vector; F.I., fold inhibition; MLV, murine leukemia virus; ZAP, zincfinger antiviral protein; ZRE, ZAP responsive element

\section{REFERENCES}

Bick, M.J., Carroll, J.W., Gao, G., Goff, S.P., Rice, C.M., and MacDonald, M.R. (2003). Expression of the zinc-finger antiviral protein inhibits alphavirus replication. J Virol 77, 11555-11562.

Chen, G., Guo, X., Lv, F., Xu, Y., and Gao, G. (2008). p72 DEAD box RNA helicase is required for optimal function of the zinc-finger antiviral protein. Proc Natl Acad Sci U S A 105, 4352- 4357.

Gao, G., Guo, X., and Goff, S.P. (2002). Inhibition of retroviral RNA production by ZAP, a CCCH-type zinc finger protein. Science 297 , 1703-1706.

Garneau, N.L., Wilusz, J., Wilusz, C.J. (2007). The highways and 
byways of mRNA decay. Nat Rev Mol Cell Biol 8, 113-126.

Guhaniyogi, J., Brewer, G. (2001). Regulation of mRNA stability in mammalian cells. Gene 265, 11-23.

Guo, X., Carroll, J.W., Macdonald, M.R., Goff, S.P., and Gao, G. (2004). The zinc finger antiviral protein directly binds to specific viral mRNAs through the $\mathrm{CCCH}$ zinc finger motifs. J Virol 78, 12781-12787.

Guo, X., Ma, J., Sun, J., and Gao, G. (2007). The zinc-finger antiviral protein recruits the RNA processing exosome to degrade the target mRNA. Proc Natl Acad Sci U S A 104, 151-156.

Jankowsky, E., and Fairman, M.E. (2007). RNA helicases-one fold for many functions. Curr Opin Struct Biol 17, 316-324.

Law, L.M., Albin, O.R., Carroll, J.W., Jones, C.T., Rice, C.M., and Macdonald, M.R. (2010). Identification of a dominant negative inhibitor of human zinc finger antiviral protein reveals a functional endogenous pool and critical homotypic interactions. J Virol 84, 4504-4512.

Liu, Q., Greimann, J.C., and Lima, C.D. (2006). Reconstitution, activities, and structure of the eukaryotic RNA exosome. Cell 127, 1223-1237.

Muller, S., Moller, P., Bick, M.J., Wurr, S., Becker, S., Gunther, S., and Kummerer, B.M. (2007). Inhibition of filovirus replication by the zinc finger antiviral protein. J Virol 81, 2391-2400.

Wang, Y., and Bogenhagen, D.F. (2006). Human mitochondrial DNA nucleoids are linked to protein folding machinery and metabolic enzymes at the mitochondrial inner membrane. J Biol Chem 281, 25791-25802.

Zhang, Y., Burke, C.W., Ryman, K.D., and Klimstra, W.B. (2007). Identification and characterization of interferon-induced proteins that inhibit alphavirus replication. J Virol 81, 11246-11255.

Zhou, Y., Ma, J., Bushan, R.B., Wu, J.Y., Pan, Q., Rong, L., and Liang, C. (2007). The packaging of human immunodeficiency virus type 1 RNA is restricted by overexpression of an RNA helicase DHX30. Virology 372, 97-106.

Zhu, Y., and Gao, G. (2008). ZAP-mediated mRNA degradation. RNA Biol 5, 65-67. 